\title{
Some Evaluations for the Generalized Hypergeometric Series
}

\author{
By J. L. Lavoie
}

\begin{abstract}
Whipple's theorem on the sum of $a_{3} F_{2}(1)$ plays a key role in obtaining a family of
\end{abstract} summation formulas for the generalized hypergeometric series of unit argument.

1. Introduction and Main Result. The object of this paper is to put on record a family of evaluation formulas for the generalized hypergeometric series of unit argument, in terms of the logarithmic derivative of the gamma function $\psi(z)=$ $d[\ln \Gamma(z)] / d z$ and the polygamma function $\psi^{(n)}(z)=d^{n} \psi(z) / d z^{n}$. These results are:

$$
{ }_{3} F_{2}\left(\begin{array}{cc}
1+c, & 1,1 \\
1+f, & 2+2 c-f
\end{array} \mid 1\right)
$$

$$
\begin{gathered}
=\frac{f(1+2 c-f)}{2 c}\left\{\psi\left(1+c-\frac{f}{2}\right)-\psi\left(\frac{1}{2}+c-\frac{f}{2}\right)\right. \\
\left.+\psi\left(\frac{1}{2}+\frac{f}{2}\right)-\psi\left(\frac{f}{2}\right)\right\}, \quad R(c)>0, \\
{ }_{4} F_{3}\left(\begin{array}{ccc}
1+a, & 1-a, 1, & 1 \\
1+f, & 2-f, & 2
\end{array}\right) \\
=\frac{f(1-f)}{a^{2}}\{\psi(1+a-f)+\psi(1-a-f)-2 \psi(1-f) \\
\quad+\frac{\sin \pi a}{2 \sin \pi f}\left[\psi\left(\frac{1}{2}+\frac{a-f}{2}\right)-\psi\left(1+\frac{a-f}{2}\right)\right. \\
\left.\left.\left.{ }_{4} F_{3}\left(\begin{array}{c}
1+c, \\
1+f, \quad 2+2 c-f, 2
\end{array}\right)+(1)+\frac{a+f}{2}\right)-\psi\left(\frac{1}{2}-\frac{a+f}{2}\right)\right]\right\},
\end{gathered}
$$

$$
\begin{aligned}
& =\frac{f(1+2 c-f)}{2 c}\left\{\psi^{(1)}(1+2 c-f)+\psi^{(1)}(f)\right. \\
& -\frac{1}{4}\left[\psi\left(1+c-\frac{f}{2}\right)-\psi\left(\frac{1}{2}+c-\frac{f}{2}\right)\right. \\
& \left.\left.+\psi\left(\frac{1}{2}+\frac{f}{2}\right)-\psi\left(\frac{f}{2}\right)\right]^{2}\right\} \text {. }
\end{aligned}
$$

Received August 21, 1984.

1980 Mathematics Subject Classification. Primary 33A35; Secondary 33A15.

Key words and phrases. Generalized hypergeometric function, gamma function, psi function, polygamma function. 
If $a \rightarrow 0$ in (2), or $c \rightarrow 0$ in (3), we find

$$
\begin{aligned}
& { }_{4} F_{3}\left(\begin{array}{c}
1,1,1,1 \\
1+f, 2-f,
\end{array} \mid 1\right) \\
& =f(1-f)\left\{\psi^{(2)}(1-f)+\frac{\pi}{2 \sin \pi f}\left[\psi^{(1)}\left(\frac{1}{2}-\frac{f}{2}\right)-\psi^{(1)}\left(1-\frac{f}{2}\right)\right]\right\} .
\end{aligned}
$$

If $c=f$ in (3), then

$$
{ }_{3} F_{2}\left(\begin{array}{ccc}
1, & 1, & 1 \\
2, & 2+f
\end{array} \mid\right)=(1+f) \psi^{(1)}(1+f) .
$$

Finally, if $f \rightarrow 0$, the last two relations reduce to the well-known result

$$
{ }_{3} F_{2}\left(\begin{array}{rrr}
1, & 1, & 1 \\
2, & 2
\end{array} \mid 1\right)=\sum_{k=1}^{\infty} k^{-2}=\zeta(2)=\frac{\pi^{2}}{6} .
$$

2. Proofs. From the elementary relation

$$
z_{p+1} F_{q+1}\left(\begin{array}{cc}
1+a_{p}, & 1 \\
1+b_{q}, & 2
\end{array} \mid z\right)=\frac{b_{1} \cdots b_{q}}{a_{1} \cdots a_{p}}\left\{{ }_{p} F_{q}\left(\begin{array}{c}
a_{p} \\
b_{q}
\end{array} \mid z\right)-1\right\}
$$

often used by Luke [2, p. 166], we write

$$
{ }_{4} F_{3}\left(\begin{array}{rrr}
1+a, & 1+r-a, & 1+c, 1 \\
1+f, & 2+2 c-f, & 2
\end{array} \mid 1\right)=\frac{f(1+2 c-f)}{a(r-a) c}\left\{w_{r}(a, c, f)-1\right\},
$$

with

$$
w_{r}(a, c, f)={ }_{3} F_{2}\left(\begin{array}{cc}
a, & r-a, c \\
f, & 1+2 c-f
\end{array} \mid 1\right) .
$$

We have

$$
w_{r}(a, c, f)=w_{r}(r-a, c, f)
$$

and the ${ }_{3} F_{2}$ can be evaluated by Whipple's theorem ([1, p. 16], or [2, p. 164]) when $r=1$. Hence

$$
w_{1}(a, c, f)=\frac{\pi \Gamma(f) \Gamma(1+2 c-f)}{2^{2 c-1} \Gamma\left(\frac{1}{2}+c+\frac{a-f}{2}\right) \Gamma\left(\frac{1}{2}-\frac{a-f}{2}\right) \Gamma\left(1+c-\frac{a+f}{2}\right) \Gamma\left(\frac{a+f}{2}\right)},
$$

$R(c)>0$. Using the simplest contiguous function relation given by Rainville [3, pp. $82,14]$, we find, in terms of $w_{1}$, that

$$
\begin{aligned}
& w_{0}(a, c, f)=\frac{1}{2} w_{1}(a, c, f)+\frac{1}{2} w_{1}(1+a, c, f), \\
& \begin{aligned}
w_{-1}(a, c, f)=\frac{1}{2(2 a+1)}\left[(a+1) w_{1}(a, c, f)+(2 a+1) w_{1}(1+a, c, f)\right. \\
\left.+a w_{1}(2+a, c, f)\right],
\end{aligned}
\end{aligned}
$$

with increasing complexity as $r=-2,-3, \ldots$ In particular, we have

(4) ${ }_{4} F_{3}\left(\begin{array}{ccc}1+a, & 2-a, 1+c, & 1 \\ 1+f, & 2+2 c-f, & 2\end{array} \mid 1\right)=\frac{f(1+2 c-f)}{a(1-a) c}\left\{w_{1}(a, c, f)-1\right\}$, 
$R(c)>0$, and

$$
\begin{aligned}
& { }_{4} F_{3}\left(\begin{array}{ccc}
1+a, & 1-a, 1+c, & 1 \\
1+f, & 2+2 c-f, & 2
\end{array} \mid 1\right) \\
& =\frac{f(1+2 c-f)}{2 a^{2} c}\left\{2-w_{1}(a, c, f)-w_{1}(1+a, c, f)\right\} .
\end{aligned}
$$

Formula (1) is obtained by letting $a \rightarrow 0$ in (4), with the aid of L'Hôpital's Rule.

Using the fact that $w_{1}(a, 0, f)=1+\sin \pi a / \sin \pi f$, we similarly find that $(2)$ is a limiting case of (5), when $c \rightarrow 0$.

Formula (3) is obtained by letting $a \rightarrow 0$ in (5) and employing L'Hospital's Rule twice. In each case, simplifications have been effected using familiar identities involving the psi and the polygamma functions.

3. A Formula of Watson. Our formula (1) is, essentially, a result given by Watson in 1917, [4], in the form

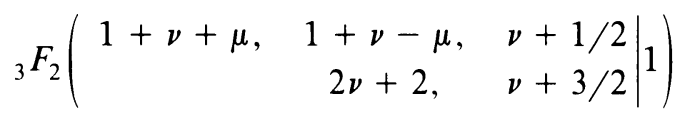

$$
\begin{aligned}
& =\frac{\Gamma(2 \nu+2)}{2 \Gamma(1+\nu+\mu) \Gamma(1+\nu-\mu)} \\
& \times\left\{\psi\left(1+\frac{\nu+\mu}{2}\right)+\psi\left(1+\frac{\nu-\mu}{2}\right)-\psi\left(\frac{1}{2}+\frac{\nu+\mu}{2}\right)\right. \\
& \left.-\psi\left(\frac{1}{2}+\frac{\nu-\mu}{2}\right)\right\} \text {. }
\end{aligned}
$$

This can be readily seen by considering a special case of a fundamental relation between ${ }_{3} F_{2}(1)[1$, p. 14]. We have

$$
\begin{aligned}
& { }_{3} F_{2}\left(\begin{array}{cc}
1+2 c-f, & f, c \\
1+2 c, & 1+c
\end{array} \mid 1\right) \\
& =\frac{c \Gamma(2 c+1)}{\Gamma(2+2 c-f) \Gamma(1+f)}{ }_{3} F_{2}\left(\begin{array}{cc}
1+c, & 1,1 \\
1+f, & 2+2 c-f
\end{array} \mid 1\right) .
\end{aligned}
$$

Hence (1) can be used on the right and (6) is obtained, after an obvious change of variables.

4. Contiguous Relations. Most of the above formulas possess contiguous relations. That is, there exist analogous formulas where some of the parameters are increased or decreased by unity. In illustration, a relation contiguous to (6) will be obtained.

With the aid of Gauss's theorem for the sum of ${ }_{2} F_{1}(1)[1$, p. 2] and two of the simplest contiguous function relations found in $[3$, pp. 84, 14, 15], we obtain a relation between two nonterminating Saalschützian series:

$$
\begin{aligned}
& { }_{3} F_{2}\left(\begin{array}{cc}
a, b, & c \\
a+b, & c+1
\end{array} \mid 1\right)=-\frac{c \Gamma(a+b)}{(a-c) \Gamma(a+1) \Gamma(b)} \\
& +\frac{a(a+b-c)}{(a+b)(a-c)}{ }_{3} F_{2}\left(\begin{array}{cc}
a+1, & b, \\
a+b+1, & c+1
\end{array} \mid 1\right) \text {. }
\end{aligned}
$$


Specializing the parameters so that (6) can be used on the right, we eventually obtain the evaluation

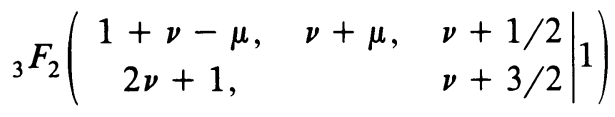

$$
\begin{aligned}
& =\frac{\Gamma(2 \nu+2)}{2(2 \mu-1) \Gamma(\nu+\mu) \Gamma(1+\nu-\mu)} \\
& \times\left\{\psi\left(\frac{\nu+\mu}{2}\right)-\psi\left(\frac{1+\nu+\mu}{2}\right)+\psi\left(1+\frac{\nu-\mu}{2}\right)-\psi\left(\frac{1+\nu-\mu}{2}\right)\right\} \text {. }
\end{aligned}
$$

Departement de Mathématiques

Université Laval

Québec, P. Q., Canada G1K 7P4

1. W. N. BaIley, Generalized Hypergeometric Series, Cambridge Univ. Press, Cambridge, 1935.

2. Y. L. LUKE, Mathematical Functions and their Approximations, Academic Press, New York, 1975.

3. E. D. RaInville, Special Functions, Macmillan, New York, 1960.

4. G. N. Watson, "The integral formula for generalized Legendre functions," Proc. London Math. Soc. (2), v. 17, 1917, pp. 241-246. 